\title{
Sistemas sensoriais no equilíbrio corporal de idosos
}

\author{
Sensory systems in the postural balance of elderly people
}

Natalia Aquaroni Ricci', Juliana Maria Gazzola², Ibsen Bellini Coimbra ${ }^{3}$

\begin{abstract}
Resumo
Os sistemas sensoriais (visual, somato-sensitivo e vestibular) são responsáveis pelo início do processo de construção do equilíbrio corporal humano. No entanto, esses sistemas sofrem alterações causadas pela senescência e pela presença de doenças. Tais modificações no funcionamento sensorial podem predispor 0 idoso à instabilidade, e, consequentemente, às quedas. 0 objetivo desta revisão de literatura foi expor os elementos sobre a função dos sistemas sensoriais no equilíbrio corporal dos idosos, dando um panorama geral da ação, avaliação e reabilitação da interação sensorial. Para estes fins, realizou-se revisão bibliográfica narrativa da literatura nacional e internacional sobre 0 tema, tendo como base textos das bases de dados Medline e Lilacs. De acordo com a literatura, existe uma grande dependência, por parte dos idosos, da informação proprioceptiva e visual para o controle postural. Contudo, nas situações de conflito entre as informações ambientais e corporais, é acionado o sistema vestibular. Entre os testes empregados na avaliação do equilíbrio em condições sensoriais podemos citar: as plataformas de força, eletromiografias, sistemas de fotofilmagens, 0 teste de Romberg e o Clinical Test of Sensory Interaction and Balance. A reabilitação do equilíbrio sensorial é baseada na exposição do idoso repetidamente às condições de dificuldade. Dentre as técnicas que podem ser empregadas na reabilitação sensorial estão o Tai Chi Chuan, a Reabilitação por Realidade Virtual e a reabilitação vestibular. Com o maior conhecimento da função dos mecanismos sensoriais no envelhecimento, é possível ao profissional da saúde detectar os déficits sensoriais e planejar as estratégias voltadas à reabilitação do equilíbrio a fim de evitar as quedas.
\end{abstract}

Palavras-chave: Idoso; equilíbrio; acidentes por quedas; interação sensorial.

\begin{abstract}
The sensory systems (visual, somatosensitive and vestibular) are responsible for the beginning of the human postural balance building process. However, these systems suffer the disorders caused by the aging process and by the presence of diseases. These modifications in the sensorial functioning may predispose the elderly to instability and, consequently, to falls. The purpose of this literature review was to expose the elements about the function of sensory systems in the postural balance of elderly people, providing a general panorama on the action, the evaluation and the rehabilitation of the sensory interaction. Thus, a bibliographic review of national and international literature on this topic was performed and carried out on Medline and Lilacs baselines. According to the literature, the essential role played by proprioceptive and visual information on elderly postural control was substantiated. However, under conflicting situations, involving both environmental and body information, the vestibular system is enabled. There are several tests utilized for balance evaluation under particular sensory conditions. Amongst such tests, we can cite the force plates, electromyography evaluations, photo shooting systems, Romberg's Test, and the Clinical Test of Sensory Interaction and Balance. Sensory balance rehabilitation is based on constant exposure of the elderly to difficult situations. Tai Chi Chuan, Rehabilitation by Virtual Reality and vestibular rehabilitation are examples of techniques used for such therapy purposes. A better understanding of the sensory systems interaction with the aging process enables health professionals to recognize sensorial deficits and, consequently, to plan strategies towards balance rehabilitation and avoid falls.
\end{abstract}

Keywords: Elderly; balance; accidental falls; sensory interaction.

Recebido: $11 / 02 / 2008$

Revisado: 29/07/2008

Aprovado: $14 / 05 / 2009$

\footnotetext{
Universidade Federal de São Carlos (UFScar), São Carlos (SP), Brasil

2 Programa de Doutorado em Ciências Otorrinolaringológicas da Universidade Federal de São Paulo (Unifesp), São Paulo (SP), Brasil

${ }^{3}$ Disciplina de Reumatologia da Faculdade de Ciências Médicas da Universidade Estadual de Campinas (FCM-Unicamp), Campinas (SP), Brasil

Endereço para correspondência:Natalia Aquaroni Ricci - Universidade Federal de São Carlos - Via Washington Luís, km 235 - CEP $13565-905$ - São

Carlos (SP), Brasil - E-mail: natalia_ricci@hotmail.com
} 


\section{Introdução}

A regulação do controle postural é dependente da relação adequada entre o corpo e as características do ambiente'. Para que esta relação ocorra, é necessária a troca de informações entre o organismo humano e o ambiente. Tal comunicação se dá, inicialmente, por meio dos sistemas sensoriais. A informação sensorial, seja qual for sua natureza, é o primeiro contato recebido pelo corpo proveniente do ambiente externo, e é a partir dela que começa o processo de construção do equilíbrio humano. À medida que o ser humano envelhece, os sistemas sensoriais responsáveis pelo controle postural são afetados pela própria diminuição da reserva funcional do idoso e/ ou pelas doenças que acometem com frequência essa faixa etária ${ }^{2}$, predispondo o indivíduo ao desequilíbrio corporal e a quedas.

Este estudo tem como objetivo fazer uma revisão sobre a função dos sistemas sensoriais no equilíbrio corporal dos idosos, dando um panorama geral da ação, avaliação e reabilitação da interação sensorial.

\section{Método}

Realizou-se revisão bibliográfica narrativa da literatura nacional e internacional sobre o tema, tendo como base textos do Medline e Lilacs, utilizando-se das palavras-chave "idoso", "equilíbrio", "reabilitação", "sistema vestibular”, "visão", "somatossensação", "interação sensorial”, "sistemas sensoriais" e/ou "quedas". Também foram verificadas as referências de livros, textos e dissertações de mestrado relevantes para a compreensão da temática. Os textos selecionados foram aqueles que abordavam diretamente o tema da pesquisa, publicados entre os anos de 1984 e 2008.

\section{Equilíbrio e quedas nos idosos}

Equilíbrio corporal e controle postural são termos utilizados como sinônimos ${ }^{3}$ e podem ser definidos como a habilidade em manter o centro de gravidade corporal projetado sobre os limites da base de sustentação durante posições estáticas e dinâmicas ${ }^{1,4-6}$.

Os limites de estabilidade durante a posição vertical estática são considerados como a angulação máxima a partir da vertical que pode ser tolerada sem a perda do controle postural' ${ }^{1}$. Para a manutenção do equilíbrio dentro dos limites de estabilidade, o sistema de controle postural tenta reposicionar o centro de gravidade por meio de oscilações corporais ou adoções de estratégias posturais.

O sistema de controle postural atua como responsável pela recuperação da estabilidade por meio de movimentos corretivos e antecipatórios escolhidos conforme o tipo e amplitude das perturbações impostas ao corpo, bem como das demandas determinadas pela tarefa e pelo ambiente ${ }^{7}$.

A instabilidade é um dos principais problemas de cuidados à saúde da população idosa ${ }^{2}$, o que se deve ao seu desfecho final: a ocorrência do evento queda.
A queda é definida como um contato não intencional com a superfície de apoio resultante da mudança de posição do indivíduo para um nível inferior à sua posição inicial, sem precedência de perda da consciência ou resultante de força externa, como um acidente inevitável ${ }^{8}$.

Aproximadamente um terço das pessoas acima de 65 anos que vivem na comunidade caem anualmente e esta proporção aumenta para $50 \%$ naqueles acima dos 80 anos?. Esses dados são semelhantes aos encontrados em estudos populacionais brasileiros em que a prevalência das quedas em idosos esteve em torno de $31^{10}$ e $35 \%{ }^{11}$. Dessas quedas, 10 a $15 \%$ resultam em danos graves (fraturas, luxações e traumas encefálicos) e 30 a 50\% têm como consequência danos menores (hematomas, arranhões, dor $)^{12}$. No entanto, mesmo as quedas que não acarretam risco de morte e injúrias trazem prejuízos à saúde do idoso. Isto porque a queda é um evento com consequências psicossociais, no qual o medo de cair novamente resulta em isolamento social e restrição nas atividades que levam à incapacidade física e mental ${ }^{13}$. Desta forma, o conhecimento de fatores relacionados ao equilíbrio que possam fazer parte do processo de reabilitação a fim de impedir as quedas, exerce um papel fundamental na restauração da capacidade funcional do idoso, reduzindo os custos causados pelas internações e incapacidades decorrentes destes eventos.

Sabe-se que os idosos que sofrem quedas não são capazes de manter o controle postural tão bem quanto aqueles que nunca caíram. No entanto, mesmo os idosos que nunca sofreram quedas frequentes admitem ter dificuldade em manter-se equilibrados e estáveis.

\section{A atuação das informações sensoriais no equilíbrio corporal}

O processamento do controle postural ocorre inicialmente pela orientação, na qual informações sobre a posição do corpo e sua trajetória no espaço são fornecidas por meio do sistema sensorial. O sistema nervoso central (SNC) recebe essas informações aferentes e seleciona respostas efetivas e reguladas no tempo para a ação estabilizadora. A execução das respostas programadas pelo SNC é feita pelo sistema efetor, composto pelo sistema musculoesquelético ${ }^{5}$.

Cada sistema sensorial oferece ao SNC informações específicas sobre a posição e o movimento do corpo; portanto, cada um deles fornece uma estrutura de referência diferente para o controle postural ${ }^{14}$. A interpretação convergente dessas informações sensoriais pelo SNC resultará na ativação do controle do alinhamento e tônus corporal em relação à gravidade, superfície de sustentação e ambiente físico ${ }^{15}$.

As informações visuais relatam dados ao SNC sobre a posição e o movimento de partes do corpo em relação às outras partes e aos objetos circunjacentes do ambiente físico ${ }^{1}$. A visão ajuda a orientar o corpo no espaço ao referenciar os eixos verticais e horizontais dos objetos ao seu redor ${ }^{5}$. As informações visuais podem ser de origem periférica, a qual consiste na capacidade de visualizar os campos laterais enquanto o olhar é dirigido à frente; e de origem central, que é também chamada de visão fóvea, que processa somente a visualização de uma pequena 
área. Os dados visuais periféricos, por serem mais amplos, são mais importantes para o controle postural ${ }^{16}$.

O sistema somato-sensitivo fornece pelos receptores articulares, tendíneos e musculares informações ao SNC em relação ao movimento do corpo no que se refere à superfície de sustentação e ao movimento dos segmentos corporais entre $\mathrm{si}^{1,17}$.

O sistema vestibular provê ao SNC informações sobre a posição e os movimentos cefálicos em relação às forças da gravidade e da inércia por meio de medidas de velocidade angular e aceleração linear da cabeça em relação ao eixo gravitacional ${ }^{1,5}$. É por meio dos canais semicirculares e órgãos otolíticos que se detectam os movimentos rotacionais e a aceleração linear da cabeça, respectivamente. O estímulo vestibular é utilizado para produzir movimentos oculares compensatórios e respostas posturais aos movimentos cefálicos ${ }^{18}$.

A contribuição destas informações sensoriais para a sensação do movimento em humanos já foi demonstrada experimentalmente, por meio da estimulação de cada sistema. Os dados somato-sensitivos foram testados por vibrações nos tendões de músculos cervicais e no tendão de Aquiles para estimular os detectores proprioceptivos ${ }^{19,20}$. Para verificar os danos causados pela ausência proprioceptiva, foi utilizado torniquete de pressão no tornozelo ${ }^{21}$. Foi utilizado o paradigma da sala móvel, que cria a ilusão da inclinação postural ${ }^{8,19}$, para testar a influência da visão na percepção do movimento. Para verificar a contribuição do sistema vestibular, foi aplicado estímulo elétrico no nervo vestibular por meio de corrente elétrica em eletrodos localizados no osso mastoide, produzindo sensações de movimento e inclinações ${ }^{19}$.

Apesar da grande utilidade dos dados multisensoriais, o SNC geralmente prioriza as informações do sistema que fornece a orientação mais adequada para o desempenho da tarefa. Contudo, ao perceber que um sistema provê informações inacuradas o SNC, por sua capacidade de adaptação, possibilita a utilização de informações alternativas de outro sistema ${ }^{6}$. Essa capacidade que o SNC tem de selecionar, em diferentes condições, os dados sensoriais mais apropriados e organizar os movimentos corporais, baseado nessas aferências, é denominada organização sensorial $^{17}$.

Geralmente os sistemas mais acionados são o somato-sensitivo e o visual, sendo que o vestibular atua principalmente quando é preciso resolver o conflito de informações sensoriais equivocadas ${ }^{6,17}$.

\section{Alterações nas informações sensoriais decorrentes do envelhecimento e doenças}

A habilidade dos sistemas sensoriais em enviar informações adequadas para o SNC pode estar comprometida pela presença de doenças, uso de medicamentos e pelo próprio processo do envelhecimento.

Pesquisas com idosos saudáveis vêm demonstrando um padrão diferente de utilização das informações sensoriais à medida que se en- velhece, demonstrando que as alterações nos sistema sensoriais decorrentes da senescência também causam modificações no equilíbrio.

Estudos revelaram que, em comparação às faixas etárias mais jovens, os idosos saudáveis são mais propensos à instabilidade quando dois dos sistemas sensoriais estão alterados ou ausentes ${ }^{4,22}$. Cohen et $a l .{ }^{23}$ verificaram a influência das informações sensoriais em diferentes faixas etárias, de jovens a idosos. Constataram, como resultado, que mudanças no equilíbrio começam na meia idade e se tornam mais pronunciadas com o avançar da idade.

Teasdale et al. ${ }^{22}$ compararam idosos saudáveis e jovens, tendo como resultado que em ambas as faixas etárias há um aumento da oscilação quando a visão é ocluída, e que os idosos são tão estáveis quanto os jovens em superfície flexível e visão normal. No entanto, quando a propriocepção está alterada (superfície flexível), a visão é removida (fechamento dos olhos) e consequentemente é usada apenas a informação vestibular, os idosos apresentam oscilações significativamente maiores do que os jovens. O sistema vestibular nos idosos não consegue, isoladamente, transmitir informações adequadas para o controle postural. Também, a visão exerce papel fundamental quando a propriocepção está inacurada.

Ao longo das décadas, a influência da visão e da propriocepção na estabilidade postural no gênero feminino foi analisada por meio da posturografia no estudo de Choy, et al. ${ }^{24}$. A avaliação revelou que a idade, a visão e a propriocepção são fatores que influenciam potencialmente no controle postural. As mulheres entre 60 e 70 anos na posição ortostática com base de sustentação confortável, superfície estável e olhos fechados apresentaram maior instabilidade quando comparadas às mulheres mais jovens, o que revela a importância da visão para o equilíbrio decorrente do envelhecimento. A propriocepção começou a exercer influência a partir dos 50 anos quando a instabilidade foi observada em posição ortostática, base de sustentação confortável, olhos abertos e superfície instável.

No estudo de Camicioli et al. ${ }^{25}$ foram comparados idosos saudáveis com diferentes faixas etárias. O resultado demonstrou que os idosos, em geral, apresentam um decréscimo no equilíbrio quando a superfície é instável, isto é quando a propriocepção está inacurada. Os idosos acima dos 80 anos tiveram um desempenho inferior estatisticamente significante em relação aos idosos mais jovens quando em superfície instável e visão normal. Esses dados revelam a dependência no sistema somatosensorial com o envelhecimento.

Os idosos também apresentam maior dificuldade em selecionar e pesar as referências para o SNC quando há incoerência ou ausência de algum dos sistemas ${ }^{26}$.

Com o envelhecimento, o sistema visual sofre uma série de mudanças, tais como: a diminuição da acuidade e do campo visual, diminuição na velocidade de adaptação ao escuro e o aumento de limiar de percepção luminosa ${ }^{27}$. Esse decréscimo na capacidade visual está associado às quedas, já que a oscilação corporal aumenta à medida que os inputs visuais diminuem em testes de equilíbrio ${ }^{4}$. 
Além das alterações próprias do envelhecimento, as moléstias oftalmológicas mais comuns nos idosos incluem a catarata, glaucoma e degeneração macular que aumentam ainda mais o risco para quedas nessa faixa etária. Lee e Scuds ${ }^{28}$ verificaram que o equilíbrio funcional de idosos com déficit visual moderado está significativamente $(p<0,001)$ mais comprometido do que em idosos sem problemas visuais.

Com o envelhecimento, o sistema somatosensorial apresenta perda de fibras sensoriais e de receptores proprioceptivos, com redução do número de corpúsculos de Pacini, Merkel e Meissner ${ }^{27}$. Essas perdas trazem prejuízos funcionais, como: a diminuição na sensação vibratória, senso de posição e sensibilidade ${ }^{4}$. Em idosos saudáveis a incapacidade ou maior dificuldade nas posições com alteração da superfície de suporte, seja por uso de espuma ou plataforma móvel, pode ser indicativo dessa diminuição na informação proprioceptiva.

Quanto às doenças que podem comprometer a propriocepção, temos: a neuropatia periférica, osteoartrite, insuficiência vascular periférica, entre outras. O estudo de Simoneau et al. ${ }^{29}$ verificou piora no desempenho e maior oscilação nos testes de equilíbrio dos portadores de neuropatia diabética quando comparados aos portadores de diabetes sem neuropatia e com grupo ausente de ambas as doenças. O déficit proprioceptivo causado pela neuropatia diminui a habilidade de manutenção da estabilidade enquanto a postura é ereta, de modo que nem o sistema visual, nem o vestibular conseguem compensar essa perda sensorial.

No envelhecimento, ocorre redução da habilidade de adaptação e compensação do sistema vestibular, provocando um processo de disfunção vestibular crônica, que pode comprometer a condição clínica e o controle postural ${ }^{30}$. O sistema vestibular sofre alterações estruturais e eletrofisiológicas com o envelhecimento, tais como: a perda das células vestibulares ciliares e nervosas, aumento do atrito das fibras nervosas do nervo vestibular, perda seletiva da densidade das fibras de mielina e a redução da velocidade de condução do estímulo elétrico no nervo vestibular ${ }^{31}$.

Quando as informações proprioceptivas e visuais estão ausentes ou inacuradas, o SNC tem o sistema vestibular como principal fonte de informação sensorial. No entanto, alguns estudos ${ }^{25,32,33}$ demonstraram que os idosos saudáveis não conseguem utilizar adequadamente os dados fornecidos pelo sistema vestibular, provavelmente pelas perdas causadas pelo envelhecimento.

As doenças que afetam o sistema vestibular são muito frequentes na população idosa, resultando frequentemente em tontura ou vertigem, sendo esses sintomas comuns em 50 a $60 \%$ dos idosos que vivem na comunidade ${ }^{34}$. Em estudo com idosos com queixa de tontura foram mais prevalentes os diagnósticos de labirintopatia metabólica, vertigem posicional paroxística benigna e labirintopatia vascular ${ }^{30}$. Os pacientes com perdas vestibulares geralmente oscilam mais e tendem a quedas nos testes de equilíbrio quando apenas a entrada de informações vestibulares está disponível.

Em estudo comparativo entre indivíduos normais e indivíduos com perda da função vestibular foi verificado que quando a proprio- cepção e a visão se tornaram deficientes ao teste a inclinação corporal dos vestibulopatas foi significantemente maior, chegando à perda total do equilíbrio quando somente o sistema vestibular estava atuante ${ }^{35}$.

Além das alterações específicas em cada sistema sensorial, ainda é possível haver perda da redundância sensorial, isto é, quando há ausência múltipla dos dados sensoriais. Outro problema é a pesagem inflexível das informações sensoriais, que é a incapacidade de selecionar o sentido adequado para o controle postural. Nesses casos os indivíduos confiam unicamente em um dos sentidos, e quando este está ausente, não ocorre a organização sensorial em busca de outras informações. Estas situações são frequentes em pacientes com déficit neurológico ${ }^{8}$.

Então, quando há um déficit ou mesmo a ausência de um sistema, consequentemente aumenta a dependência nos sistemas remanescentes. Como os três sistemas sensoriais sofrem alterações com o processo de envelhecimento normal e devido às doenças, como já exposto, a redundância de informações se torna maior. Também, a escolha da informação sensorial pelo SNC pode ser insuficiente para produzir uma resposta rápida e correta em muitas situações que envolvem tarefas complexas e ambientes indesejáveis ${ }^{36}$.

A informação sensorial assume uma função fundamental no controle postural dos idosos, e a integração dessas informações visuais, proprioceptivas e vestibulares tornam-se necessárias para gerar respostas apropriadas para a manutenção do equilíbrio ${ }^{37}$. Assim, fica claro que a inabilidade na recepção dos elementos sensoriais múltiplos pode ser um fator contribuinte para a instabilidade e, consequentemente, um forte risco de quedas.

\section{Avaliação das informações sensoriais no equilíbrio corporal}

Devido ao caráter debilitante das quedas para compreender e possibilitar a redução na frequência e nos danos causados por elas se faz necessário identificar quais mudanças ocorrem no sistema do controle postural com o processo do envelhecimento ${ }^{38}$, bem como o motivo pelo qual os idosos falham ao tentar recuperar a estabilidade frente a este evento.

Para verificar o acometimento nos sistemas que agem no controle postural, existem testes funcionais e laboratoriais que simulam, justamente, as demandas envolvidas na habilidade em controlar o equilíbrio. Esses testes podem ser úteis para gerar hipóteses quanto aos determinantes da limitação funcional observada ${ }^{39}$ e também como forma de rastreio na identificação dos sujeitos que apresentam risco de quedas.

Vale ressaltar que os testes funcionais e laboratoriais são menos sensíveis e preditivos para idosos que tenham menos problemas de saúde e sejam independentes funcionalmente. Assim, a utilidade de um teste prever o risco de quedas e revelar déficits de equilíbrio é dependente do status de saúde e nível funcional da população a ser testada ${ }^{37}$. 
Entre os testes laboratoriais empregados na avaliação do equilíbrio em condições sensoriais, temos as plataformas de força, eletromiografias e sistemas de fotofilmagens. Essas avaliações medem principalmente as oscilações corporais, deslocamento do centro de gravidade e ativação muscular.

A avaliação da integração sensorial e o controle dinâmico, pelos protocolos de posturografia e laboratoriais, são minuciosos e oferecem grande precisão para a detecção dos componentes comprometidos do equilíbrio ${ }^{40}$. No entanto, esses sistemas são muito sofisticados, possuem alto custo para sua implantação e uso, além de necessitar de treinamento especializado dos avaliadores e requerer espaço. Tais fatores podem limitar o uso desses sistemas no ambiente clínico ${ }^{32}$.

Existem também os testes funcionais de equilíbrio, que apesar de não terem a confiabilidade da metodologia quantitativa, têm grande efetividade na compreensão do desempenho funcional dos ajustes posturais. De forma simples e com baixo custo, podem evidenciar alterações que impliquem, na prática, em uma intervenção terapêutica direcionada à melhora da capacidade funcional ${ }^{30}$.

Diversos testes funcionais em posição estática envolvendo alterações no input visual, mudanças na superfície de sustentação e redução da base de sustentação são aplicados a fim de manipular as entradas sensoriais, e com isso, possibilitar a compreensão da ação sensorial no controle postural. O primeiro teste de equilíbrio estático foi o teste de Romberg, no qual o paciente fica em posição ortostática, pés unidos, braços ao longo do corpo e olhos abertos. À medida que os estudos avançaram, versões sensibilizadas do teste de Romberg foram propostas, tais como: fechar os olhos, posição tandem e apoio unipodal ${ }^{41}$. A avaliação é feita por meio da duração de tempo em cada posição e pela observação das posições, isto é, a quantidade de oscilação e estratégias desenvolvidas para a manutenção do equilíbrio.

Com esta mesma finalidade e com a intenção de padronizar a avaliação sensorial foi criado por Shumway-Cook e Horak ${ }^{6}$ o Clinical Test of Sensory Interaction and Balance (CTSIB) também conhecido como Teste de Interação Sensorial. O CTSIB é a versão para o meio clínico do protocolo de posturografia dinâmica das condições sensoriais, também denominado de Sensory Organization Test (SOT). Segundo El-Kashlan et al. ${ }^{42}$ o CTSIB apresenta boa correlação com a posturografia e pode ser utilizado como instrumento de rastreio na identificação de indivíduos com déficits no controle postural.

O CTSIB fornece informações sobre a capacidade do indivíduo de adaptação e manutenção do equilíbrio frente aos conflitos sensoriais impostos pelo teste, além de revelar o sistema sensorial no qual o indivíduo é mais dependente para o controle postural ${ }^{6}$.

O teste consiste no posicionamento do sujeito em posição ortostática com os braços ao longo do corpo sob seis condições intersensoriais, com a meta de permanência de 30 segundos em cada posição. $\mathrm{Na}$ condição 1 (olhos abertos em superfície firme) todos os sentidos estão presentes; na condição 2 (olhos fechados em superfície firme) o sistema visual não oferece informações; na condição 3 (cúpula visual em superfície firme) há conflito da informação visual; na condição 4 (olhos abertos em superfície de espuma) há informações inacuradas do sistema somato-sensitivo; na condição 5 (olhos fechados em superfície de espuma) há informações inacuradas do sistema somato-sensitivo e ausência do sistema visual; na condição 6 (cúpula visual em superfície de espuma) há informações inacuradas do sistema somato-sensitivo e conflito do sistema visual.

A pontuação pode ser feita por classificação categórica (normal/ anormal) e intervalar (tempo), sendo medido o tempo despendido em cada condição do CTSIB.

O CTSIB e seu uso laboratorial por meio do SOT vêm sendo utilizados na prática clínica e em pesquisa por fisioterapeutas, terapeutas ocupacionais e clínicos para avaliar e monitorar pessoas com risco de quedas $^{37}$, após acidente vascular encefálico ${ }^{43}$, com déficit vestibular $^{32} \mathrm{e}$ diabetes mellitus ${ }^{39}$, entre outros.

\section{Reabilitação da informação sensorial no equilíbrio corporal}

A partir dos resultados da avaliação é possível planejar o processo de reabilitação voltado à melhora do equilíbrio por meio das informações sensoriais remanescentes.

Como as alterações nos sistemas sensoriais variam entre os indivíduos e de acordo com a faixa etária, o treinamento do controle postural deve ser mutável conforme a necessidade de cada paciente. A reabilitação padronizada pode ser benéfica para os jovens, que possuem diferenças mínimas entre si e poucas doenças, mas para os idosos é ineficiente devido à variabilidade interpessoal e à presença de comorbidades $\operatorname{associadas}^{23}$.

Expor o idoso repetidamente às condições de dificuldade pode gerar um aprendizado da tarefa e melhora nos resultados. Também é possível treinar a confiança nos demais sistemas e diminuir a dependência em um único sistema sensorial.

Para a reabilitação sensorial, as mesmas posições propostas pelos testes de avaliação podem ser utilizadas, aumentando gradativamente o nível de dificuldade e complexidade dessas posições. Assim, podem ser propostas para a terapia: aumentar o tempo em cada posição, utilizar espumas de densidades e materiais diferentes, posicionar a cabeça em diferentes angulações, usar diferentes conflitos visuais, variar a base de sustentação, entre outros recursos.

$\mathrm{O}$ treinamento da orientação sensorial foi proposto por $\mathrm{Hu}$ e Woollacott ${ }^{44,45}$ por meio de um protocolo terapêutico que se concentrou no uso de informações sensoriais diferentes e na integração desses dados em condições sensoriais alteradas ou inexistentes. Para verificar o efeito da reabilitação sensorial, os autores compararam dois grupos de idosos. Um grupo foi submetido ao treinamento durante duas semanas, com sessões de cinco vezes por semana e duração de uma hora cada e o outro não recebeu nenhum tipo de tratamento. Os 
resultados demonstraram que os idosos que passaram pelo processo de reabilitação apresentam melhora significante em relação ao grupo controle para as condições em que a informação proprioceptiva estava alterada e quando dois sistemas estavam sendo manipulados simultaneamente, isto é, nas condições mais desafiadoras. Além disso, após um mês do treinamento, esses idosos conseguiram permanecer mais tempo em apoio unipodal e caíram com frequência menor nos testes que aboliam a somatossensaçã ${ }^{44}$. $\mathrm{Na}$ análise eletromiográfica, foi verificado que o grupo de treinamento apresentava após o final da terapia um efeito otimizado da musculatura e, consequentemente, nas respostas posturais ${ }^{45}$.

Uma modalidade que se destaca no treino de equilíbrio e redução de quedas em idosos é o Tai Chi Chuan. A técnica oriental resultou em melhora da interação sensorial nos praticantes idosos com redução do conflito sensorial nas tarefas que exigem maior contribuição do sistema vestibular e visual, aumento da propriocepção na articulação do joelho e melhora do controle postural em posição estática utilizando somente informações vestibulares ${ }^{46}$.

Uma técnica recente que contribui para o treino sensorial é a reabilitação por realidade virtual (RRV). A RRV consiste na reprodução da tarefa a ser desempenhada pelo paciente em interação com um ambiente multidimensional e multisensorial criado por computador que pode ser explorado em tempo real ${ }^{47}$.

Idosos com desordens do equilíbrio corporal foram submetidos a um protocolo de RRV com diferentes condições sensoriais, simulan- do estímulos ambientais. Houve diminuição significante dos valores da velocidade de oscilação e da área de deslocamento do centro de massa após seis semanas de treinamento ${ }^{48}$.

Sendo assim, é possível dizer que a reabilitação voltada às informações sensoriais traz benefícios para a população idosa e deve ser instaurada quando forem detectados déficits na orientação sensorial.

Para os déficits sensoriais vestibulares, existe também a possibilidade de tratamento específico por meio da reabilitação vestibular (RV). A RV representa valiosa opção terapêutica para os distúrbios do equilíbrio corporal de origem vestibular, atuando por meio de mecanismos centrais de neuroplasticidade (adaptação, habituação e substituição) para obtenção da compensação vestibular ${ }^{49}$.

\section{Considerações finais}

Os dados sensoriais são primordiais para o equilíbrio humano, visto que são as portas de entrada para todo um conjunto de reações que visam à estabilidade corporal. É preciso atenção por parte do profissional da saúde às doenças que podem comprometer a função dos sistemas sensoriais. Além disso, é preciso incorporar, na rotina clínica, testes para detectar déficits sensoriais que resultam na instabilidade corporal. Com esses cuidados é possível prevenir o comprometimento na função dos sistemas sensoriais em idosos e reabilitar precocemente, quando necessário, os sistemas deficitários. Assim, será possível reduzir a instabilidade postural e as quedas.

\section{Referências}

1. Shumway-Cook A, Woollacott MH. Controle postural normal. In: ShumwayCook A, Woollacott MH. Controle Motor - teoria e aplicações práticas. $2^{\mathrm{a}}$ ed. Barueri: Manole; 2003. p. 153-78.

2. Konrad HR, Girardi M, Helfert R. Balance and aging. Laryngoscope 1999;109(9):1454-60.

3. Ragnarsdóttir M. The concept of balance. Physiotheraphy 1996;82(6): $368-75$.

4. Alexander NB. Postural control in older adults. J Am Geriatr Soc 1994;42(1):93-108.

5. Chandler JM. Equilíbrio e Quedas no Idoso: Questões sobre a Avaliação e o Tratamento. In: Guccione AA. Fisioterapia Geriátrica. $2^{2}$ ed. Rio de Janeiro: Guanabara Koogan; 2002. p. 265-77.

6. Shumway-Cook A, Horak FB. Assessing the influence of sensory interaction on balance. Suggestion from the field. Phys Ther 1986;66(10):1548-50.

7. Huxham FE, Goldie PA, Patla AE. Theoretical considerations in balance assessment. Aust J Physiother 2001;47(2):89-100.

8. Shumway-Cook A, Woollacott MH. Envelhecimento e controle postural. In: Shumway-Cook A, Woollacott MH. Controle Motor - teoria e aplicações práticas. $2^{\mathrm{a}}$ ed. Barueri: Manole; 2003. p. 209-31.
9. O'loughlin JL, Robitaille Y, Boivim JF, Suissa S. Incidence of and risk factors for falls and injurious falls among the community-dwelling elderly. Am J Epidemiol 1993;137(3):342-54.

10. Perracini MR, Ramos LR. Fatores associados a quedas em uma coorte de idosos residentes na comunidade. Rev Saúde Pública 2002;36(6):709-16.

11. Siqueira FV, Facchini LA, Piccini RX, Tomasi E, Thumé E, Silveira DS et al. Prevalência de quedas em idosos e fatores associados. Rev Saúde Pública 2007:41(5):749-56.

12. Nevitt MC. Falls in the elderly: risk factors and prevention. In: Masdeu JC Sudarsky L, Wolfson L, editors. Gait disorders of aging. Falls and Therapeutic strategies. Lippincott-Raven Publishers; 1997. p. 13-36.

13. Stalenhoef PA, Diederiks JPM, Knottnerus JA, Kester ADM, Crebolder HFJM. A risk model for the prediction of recurrent falls in community-dwelling elderly: a prospective cohort study. J Clin Epidemiol 2002;55(11):1088-94.

14. Gurfinkel VS, Levick YS. Perceptual and automatic aspects of the postural body scheme. In: Paillard, J. Brain and Space. New York: Oxford Science, 1991.

15. Horak FB. Postural orientation and equilibrium: what do we need to know about neural control of balance to prevent falls? Age Ageing 2006; 35(Suppl 2):7-11. 
16. Paulus WM, Straube A, Brandt TH. Visual stabilization of posture: physiological stimulus characteristics and clinical aspects. Brain 1984; 107(Pt 4):1143-63.

17. Hobeika CP. Equilibrium and balance in the elderly. Ear Nose Throat J 1999; 78(8):558-66

18. Carpenter MG, Frank JS, Silcher CP, Peysar GW. The influence of postural threat on the control of upright stance. Exp Brain Res 2001;138(2):210-8.

19. Horak FB, Shupert C. Função do sistema vestibular no controle postural. In: Herdman SJ. Reabilitação Vestibular. São Paulo: Manole; 2002. p. 490-504.

20. Nakagawa H, Ohashi N, Watanabe $Y$, Mizukoshi K. The contribution of proprioception to posture control in normal subjects. Acta Otolaryngol Suppl 1993;504:112-6.

21. Diener HC, Dichgan J, Gushulbauer B, Mau H. The significance of proprioception on postural stabilization as assessed by ischemia. Brain Res 1984;296(1):103-9.

22. Teasdale N, Stelmach G, Breunig A, Meeuwsen H. Age differences in visual sensory integration. Exp Brain Res 1991;85(3):691-6.

23. Cohen H, Heaton LG, Congdon SL, Jenkins HA. Changes in sensory organization test scores with age. Age Ageing 1996;25(1):39-44

24. Choy NL, Brauer S, Nitz J. Changes in postural stability in women aged 20 to 80 years. J Gerontol A Biol Sci Med Sci 2003;58(6):525-30.

25. Camicioli R, Panzer VP, Kaye J. Balance in the healthy elderly: posturography and clinical assessment. Arch Neurol 1997;54(8):976-81.

26. Horak FB, Shupert CL, Mirka A. Components of postural dyscontrol in the elderly: a review. Neurobiol Aging 1989;10(6):727-38.

27. Garcia DM, Taveira RF. Reeducação cinesioterápica das alterações do equilíbrio no processo de envelhecimento fisiológico. [Dissertação de mestrado]. Minas Gerais: Universidade Federal de Minas Gerais; 1998.

28. Lee HKM, Scudds RJ. Comparison of balance in older people with and without visual impairment. Age Ageing 2003;32(6):643-9.

29. Simoneau GG, Ulbrecht JS, Derr JA, Cavanagh PR. Role of somatosensory input in the control of human posture. Gait Posture 1995;3:115-22.

30. Gazzola JM, Ganança FF, Aratani MC, Ganança MM. Fatores associados ao equilíbrio funcional em idosos com disfunção vestibular crônica. Rev Bras Otorrinolaringol 2006;72(5):683-90

31. Sloane PD, Baloh RW, Honrubia V. The vestibular system in the elderly: clinical implications. Am J Otolaryngol 1989;10(6):422-9.

32. Cohen H, Blatchly CA, Gombash LL. A study of the clinical test of sensory interaction and balance. Phys Ther 1993;73(6):346-51.

33. Manchester D, Woollacott M, Hylton NZ, Marin O. Visual, vestibular and somatosensory contributions to balance control in the older adult. $J$ Gerontol 1989; 44(4): M118-127.
34. Ganança MM, Caovilla HH. A vertigem e sintomas associados. In: Ganança MM, Vieira RM, Caovilla HH. Princípios de otoneurologia. São Paulo: Atheneu; 1998. p. 3-5.

35. Horak F, Nashner L, Diener H. Postural strategies associated with somatosensory and vestibular loss. Exp Brain Res 1990;82(1): 167-77.

36. Perracini MR. Equilíbrio e controle postural em idosos. Rev Bras de Postura e Movimento 1998;2(4):130-42.

37. Anacker SL, Di Fabio RP. Influence of sensory inputs on standing balance in community-dwelling elders with a recent history of falling. Phys Ther 1992;72(8):575-83.

38. Hageman PA, Leibowitz M, Blanke D. Age and gender effects on postural control measures. Arch Phys Med Rehabil 1995;76(10): 961-5.

39. Cordeiro RC. Caracterização clínico-funcional do equilíbrio em idosos portadores de Diabetes Mellitus do tipo II. Tese [Mestrado]. Escola Paulista de Medicina da Universidade Federal de São Paulo: 2001.

40. El-Kashlan HK, Shepard NT, Asher AM, Smith-Wheelock M, Telian SA. Evaluation of clinical measures of equilibrium. Laryngoscope 1998;108(3):311-9.

41. Jbabdi M, Boissy P, Hamel M. Assessing control of postural stability in community-living older adults using performance-based limits of stability. BMC Geriatr 2008;8:8.

42. Lanska DJ, Goetz CG. Romberg's sign: development, adoption and adaptation in the 19th century. Neurology 2000;55(8):1201-6.

43. Di Fabio RP, Badke MB. Relationship of sensory organization to balance function in patients with hemiplegia. Phys Ther 1990;70(9):542-8.

44. $\mathrm{Hu} \mathrm{MH}$, Woollacott MH. Multisensory training of standing balance in older adults: 1. Postural stability and one-leg stance balance. J Gerontol 1994;49(2):M52-61.

45. Hu MH, Woollacott MH. Multisensory training of standing balance in older adults: 2. Kinematic and electromyographic posturl responses. J Gerontol 1994;49(2):M62-71.

46. Tsang WW, Hui-Chan CW. Sensorimotor control of balance: a Tai Chi solution for balance disorders in older subjects. Med Sport Sci 2008;52: 104-14.

47. Sveistrup H. Motor rehabilitation using virtual reality. J Neuroeng Rehabil 2004;10(1):1-10.

48. Suárez H, Suárez A, Lavinsky L. Postural adaptation in elderly patients with instability and risk of falling after balance training using a virtual-reality system. Int Tinnitus J 2006;12(1):41-4.

49. Ganança FF, Perracini MR, Ganança CF. Reabilitação dos distúrbios do equilíbrio corporal. In: Ganança MM. Vertigem: abordagens diagnósticas e terapêuticas. São Paulo: Lemos; 2002. p. 16. 